\title{
SARS-CoV-2 Re-Infection versus Prolonged Shedding: A Case Series
}

Erin Nicholson ${ }^{1}$, Vasanthi Avadhanula ${ }^{1}$, Sonia Fragoso ${ }^{1}$, Rachel Stroh ${ }^{1}$, Xunyan $\mathrm{Ye}^{1}$, Nanette Bond ${ }^{1}$, Patricia Santarcangelo ${ }^{1}$, John Stroh $^{2}$, and Pedro Piedra ${ }^{1}$

${ }^{1}$ Baylor College of Medicine

${ }^{2}$ CHI St Luke's Health

May 16, 2021

\begin{abstract}
Since the start of the SARS-CoV-2 pandemic, it has been difficult to differentiate between SARS-CoV-2 re-infection and prolonged RNA shedding. In this report, we identified patients with positive rtPCR results for SARS-CoV-2 [?]70 days apart. Clinical and laboratory data were collected and criteria were applied to discern whether the presentation was consistent with SARS-CoV-2 re-infection or prolonged viral RNA shedding. Eleven individuals met the initial testing criteria, of which, seven met at least one criteria for re-infection and four were consistent with prolonged RNA shedding. These data demonstrate the need for criteria to differentiate SARS-CoV-2 re-infection from prolonged RNA shedding.
\end{abstract}

\section{SARS-CoV-2 Re-Infection versus Prolonged Shedding: A Case Series}

Erin G. Nicholson, M.D. ${ }^{1,2}$ Vasanthi Avadhanula, Ph.D. ${ }^{1}$ Sonia Fragoso, M.P.H. ${ }^{1}$ Rachel Stroh ${ }^{3}$ Xunyan Ye, Ph.D. ${ }^{1}$ Nanette Bond, P.A. ${ }^{1}$ Patricia Santarcangelo, R.N. ${ }^{1}$ John Stroh, M.D. ${ }^{4}$ Pedro A Piedra, M.D. ${ }^{1,2}$

Corresponding Author:

Erin G. Nicholson, MD

Phone Number: 713-798-8240

Fax Number: 713-798-8250

Email Address: Erin.Nicholson@bcm.edu

Running Heading: SARSCoV2 Re-Infection/Prolonged Shedding

${ }^{1}$ Department of Molecular Virology and Microbiology, Baylor College of Medicine, Houston, TX, 77030, United States of America.

${ }^{2}$ Department of Pediatrics, Baylor College of Medicine, Houston, TX, 77030, United States of America

${ }^{3}$ School of Medicine, Baylor College of Medicine, Houston, TX, 77030, United States of America

${ }^{4}$ Department of Emergency Medicine, St. Luke's Health, Houston, TX, 77030, United States of America

Manuscript Word Count: 149411The authors have nothing to disclose and do not have any financial relationships relevant to the research discussed. Internal funds from Baylor College of Medicine were used to support this work.' 22Internal funds from Baylor College of Medicine were used to support this work.' 33 None of the Author's affiliations have changed since the completion of this study. These data have not been presented 
at a meeting to date. Corresponding Author: Erin G. Nicholson, MD Phone Number: 713-798-8240 Fax Number: 713-798-8250 Email Address: Erin.Nicholson@bcm.edu

\section{SARS-CoV-2 Re-Infection versus Prolonged Shedding: A Case Series}

Authors: Erin Nicholson, MD, Vasanthi Avadhanula, PhD, Sonia Fragoso, MPH, Rachel Stroh, Xunyan Ye, PhD, Nanette Bond, PA, Patricia Santarcangelo, RN, John Stroh, MD, Pedro A Piedra, MD

Since the start of the SARS-CoV-2 pandemic, it has been difficult to differentiate between SARS-CoV-2 re-infection and prolonged RNA shedding. In this report, we identified patients with positive rtPCR results for SARS-CoV-2 [?]70 days apart. Clinical and laboratory data were collected and criteria were applied to discern whether the presentation was consistent with SARS-CoV-2 re-infection or prolonged viral RNA shedding. Eleven individuals met the initial testing criteria, of which, seven met at least one criteria for re-infection and four were consistent with prolonged RNA shedding. These data demonstrate the need for criteria to differentiate SARS-CoV-2 re-infection from prolonged RNA shedding.

Key Words: SARS-CoV-2; Re-Infection; Shedding

Abstract Word Count: 100

\section{Background}

Since the beginning of the severe acute respiratory syndrome coronavirus-2 (SARS-CoV-2) pandemic, the potential for re-infection with the SARS-CoV-2 virus has been a concern. Endemic coronaviruses are known to re-infect individuals in the same year, so it is plausible that natural infection with SARS-CoV-2 would similarly provide short-lived immunity. However, due to prolonged viral RNA shedding of the SARS-CoV-2 virus, it has been difficult to differentiate prolonged shedding from true re-infection. ${ }^{1}$ This has changed in recent months when several reports emerged of individuals re-infected with genetically different lineages of SARS-CoV-2. ${ }^{2,3}$ These reports represent confirmation that, while uncommon, re-infection in the span of several months with SARS-CoV-2 can occur.

Currently, the Center for Disease Control (CDC) does not recommend testing individuals for re-infection less than 90 days since symptom onset. ${ }^{4,5}$ However, detailed SARS-CoV-2 real-time reverse transcriptase polymerase chain reaction (rtPCR) kinetics data appear to describe a cessation of viral RNA shedding after 70 days in the majority of individuals (though exceptions have been described). ${ }^{6-8}$ In this brief report, we describe the clinical characteristics and laboratory results of individuals with multiple positive SARS-CoV-2 rtPCR results that extend beyond 70 days.

\section{Methods}

The data described were obtained from two sources. The first is from our diagnostic operation for SARS-CoV2 testing in a Clinical Laboratory Improvement Amendments (CLIA) Certified Respiratory Virus Diagnostic Laboratory (ID\#: 45D0919666) that began on March 18, 2020. ${ }^{9}$ The second source came from rtPCR positive SARS-CoV-2 patients who enrolled in our ongoing study to evaluate antibody response kinetics after their primary infection. We included patients with 2 or more positive rtPCR tests for SARS-CoV-2 that were at least 70 days apart in order to fully capture a spectrum of patients with prolonged viral RNA shedding vs re-infection.

Based on proposed re-infection criteria in the literature, we defined three separate criteria to classify a patient as having a re-infection with SARS-CoV-2. These criteria are (1) a positive SARS-CoV-2 rtPCR test at least 90 days after individuals first became symptomatic with SARS-CoV-2 and subsequently become asymptomatic, ${ }^{4}(2)$ at least 2 negative rtPCR tests for SARS-CoV-2 between two positive tests, ${ }^{10,11}$ and (3) at least one negative rtPCR test for SARS-CoV-2 between two positive tests more than 28 days apart. ${ }^{12}$ For our analysis, only one criterion was needed to be considered a probable re-infection.

We collected demographic, laboratory, and clinical data from retrospective chart review as well as performed phone interviews using a structured questionnaire. The protocol was approved by the institutional review 
board at Baylor College of Medicine.

rtPCR Assays

Mid-turbinate swabs were tested for SARS-CoV-2 using CDC's EUA for CDC 2019-Novel Coronavirus (2019-nCoV) rtPCR Diagnostic Panel. (Primers and probes target the nucleocapsid (N) gene (N1 and N2) of SARS-CoV-2 and ribonuclease P (RNase-P) gene). The samples were considered positive if N1, N2, and RNaseP cycle threshold $(\mathrm{CT})$ values were $<40 .^{9}$

\section{IgG Assay}

The humoral IgG anti-spike enzyme linked immunosorbent assays (ELISA) were performed as part of our IRB approved research study. The SARS-CoV-2 full spike (S) protein used in the ELISA was kindly provided by Gale Smith (Novavax, Gaithersburg, MD). ${ }^{13}$ The samples were considered serologically positive if the Optical Density (OD) values were [?]0.5 at a serum dilution of $>1: 1024$.

\section{Results}

Case Series

Our laboratory tested 17,784 samples (from 13,257 patients) for SARS-CoV-2 via rtPCR from March $18^{\text {th }}$, 2020 to October $31^{\text {st }}, 2020$. Of those, 1,566 samples (from 981 patients) were positive. Within this cohort, we found 11 patients that had at least two positive SARS-CoV-2 rtPCR tests that spanned $>70$ days.

Clinical Presentation

Of these 11 individuals, 6 were female with an average age of 49 years (Table 1 ). The majority of individuals ( 8 of 11) had at least 1 co-morbid condition. All patients were symptomatic prior to and at their first positive rtPCR test. Symptom durations (when available) ranged from 4-28 days. Overall, symptoms were mild and none were hospitalized. Only 1 individual presented to the ED for shortness of breath. The majority of rtPCR testing performed after 70 days was performed either as clearance for a procedure or as part of a surveillance program.

Only two individuals were symptomatic at the time of their testing $>70$ days after initial symptoms with their primary SARS-CoV-2 infection. Both individuals had complete resolution of symptoms with their primary infection.

Probable Re-Infection Definition

We used three criteria to assess the likelihood of a SARS-CoV-2 re-infection that occurred greater than 70 days after primary infection (described above). We found that 7 of the 11 individuals met at least one criterion for probable re-infection and all 7 of these individuals met Criteria 1 (see Figure 1 and Table 1). Three (patients 1-3) of the 7 individuals with probable re-infection had at least one negative test prior to another SARS-CoV-2 rtPCR positive test, thus fitting Criteria 2 or 3 . Viral sequencing was performed, however, none of the samples $>70$ days out had sufficient genomic material for whole genome sequencing (WGS) or Sanger sequencing (data not shown). Four (patients 8-11) individuals did not meet any of the criteria for re-infection and were considered to be experiencing prolonged shedding of SARS-CoV-2 RNA.

\section{Serology}

Six of the 11 individuals had IgG serology testing for SARS-CoV-2 (Table 1). Only two individuals (patients 1 and 3) had a paired sample whose sera was collected before and after their probable re-infection. Neither of these individuals mounted an increase in IgG anti-S antibody levels after their probable re-infection (Table $1)$.

\section{Discussion}

In this case series, we identified 11 individuals who had viral RNA detected from their upper respiratory tract $>70$ days after their primary SARS-CoV-2 infection. We describe two groups: a probable SARS- 
CoV-2 re-infection group and a probable primary infection group with prolonged viral RNA shedding. Of these 11 individuals, 7 were consistent with probable re-infection, while 4 did not meet the criteria for probable re-infection and thus were considered primary infection with prolonged viral RNA shedding. We based this conclusion on several factors. First, two patients had [?]2 negative rtPCR tests prior to their probable SARS-CoV-2 re-infection and one patient (patient 3) had 1 negative test prior to their probable re-infection. Second, all 7 individuals had at least 90 days between their primary SARS-CoV-2 infection and their probable SARS-CoV-2 re-infection. We attempted to be more inclusive for re-infections occurring $<90$ days after initial symptom onset with primary infection, however, none were identified. Our data support the assertion by the CDC that re-infection is unlikely to occur within 90 days from onset of symptoms with primary SARS-CoV-2 infection. ${ }^{4,5}$

Definitive SARS-CoV-2 re-infection has been confirmed in a small number of cases. ${ }^{2,3}$ Confirmation via WGS demonstrated that the SARS-CoV-2 isolate detected with the re-infection event was sufficiently different from the isolate sequenced from the primary infection. In our population, we were unable to confirm SARS-CoV-2 re-infection cases using WGS or Sanger sequencing of the S gene because the viral RNA quantity isolated during the probable re-infection events was insufficient despite multiple sequencing attempts. In two cases, we had blood samples collected prior to and after their probable SARS-CoV-2 re-infection. In both cases, we did not observe a four-fold or greater rise in IgG anti-S antibody levels consistent with an infection. Lack of a significant antibody rise has been reported in another case report with confirmed SARS-CoV-2 infection. ${ }^{2,14}$ This lack of antibody response with re-infection was attributed to the patient's mild illness, which is consistent with our observation that both of our patients were asymptomatic with their re-infection event. ${ }^{14}$

Early re-infection or prolonged viral RNA shedding with primary SARS-CoV-2 infection might be a manifestation of non-sterilizing immunity that is generated with the initial infection. Unlike other limited case reports, which have documented recurrence of infections after hospitalization due to SARS-CoV-2, this is an ambulatory population with relatively mild symptomatology. ${ }^{12}$ None of our 7 patients were hospitalized during their first episode and only 2 patients were symptomatic with their probable re-infection event. Patient 5 had the highest severity during her probable re-infection, and she was also the oldest patient in our cohort. Another study that examined viral kinetics of SARS-CoV-2 found that a higher severity of symptoms (ICU admission) significantly decreased a patient's likelihood of prolonged shedding of SARS-CoV-2 RNA. ${ }^{6}$ This coupled with observations that individuals with severe cases of SARS-CoV-2 infection have increased IgG anti-S antibody responses compared with mild cases could explain why we see predominantly mild presentations in our cohort resulting in probable re-infections or primary infection with prolonged viral RNA shedding. ${ }^{13,15}$

\section{Limitations}

This was a single site cohort study that reported on approximately 7 months of rtPCR testing for SARSCoV-2. The low viral RNA content in the samples collected from probable re-infection cases precluded our ability to confirm the re-infection event using sequencing.

\section{Conclusions}

The occurrence of probable re-infection with SARS-CoV-2 in our population seemed to be an uncommon occurrence that was associated with mild symptomatology at the time of their primary infection. Our data does support the CDC's recommendation that testing for SARS-CoV-2 re-infection is not needed prior to 90 days after symptoms arise.

\section{References:}

1. Chen $\mathrm{W}, \mathrm{Hu} \mathrm{Z}$, Yi C, et al. An unusual COVID-19 case with over four months of viral shedding in the presence of low neutralizing antibodies: a case report. J Biomed Res . 2020;34(6):470. doi:10.7555/jbr.34.20200099

2. To KK-W, Hung IF-N, Ip JD, et al. Coronavirus Disease 2019 (COVID-19) Re-infection by a Phyloge- 
netically Distinct Severe Acute Respiratory Syndrome Coronavirus 2 Strain Confirmed by Whole Genome Sequencing. Clin Infect Dis . August 2020. doi:10.1093/cid/ciaa1275

3. Colson P, Finaud M, Levy N, Lagier JC, Raoult D. Evidence of SARS-CoV-2 re-infection with a different genotype. J Infect . 2020. doi:10.1016/j.jinf.2020.11.011

4. Overview of Testing for SARS-CoV-2 (COVID-19) | CDC.Natl Cent Immun Respir Dis (NCIRD), Div Viral Dis . 2020. https://www.cdc.gov/coronavirus/2019-ncov/hcp/testing-overview.html. Accessed December 9, 2020.

5. Duration of Isolation and Precautions for Adults with COVID-19 | CDC. https://www.cdc.gov/coronavirus/2019-ncov/hcp/duration-isolation.html. Published 2020. Accessed December 9, 2020.

6. Huang J, Zheng L, Li Z, et al. Kinetics of SARS-CoV-2 positivity of infected and recovered patients from a single center. Sci Rep . 2020;10(1). doi:10.1038/s41598-020-75629-x

7. Madhi SA, Polack FP, Piedra PA, et al. Respiratory Syncytial Virus Vaccination during Pregnancy and Effects in Infants. N Engl J Med . 2020;383(5):426-439. doi:10.1056/NEJMoa1908380

8. Wang J, Hang X, Wei B, et al. Persistent SARS-COV-2 RNA positivity in a patient for 92 days after disease onset: A case report. Medicine (Baltimore) . 2020;99(34):e21865. doi:10.1097/MD.0000000000021865

9. Avadhanula V, Nicholson E, Ferlic-Stark L, et al. Viral load of SARS-CoV-2 in adults during the first and second wave of COVID-19 pandemic in Houston, TX: the potential of the super-spreader. J Infect Dis . 2021; (Accepted).

10. Liang C, Cao JT, Liu Z, et al. Positive RT-PCR test results after consecutively negative results in patients with COVID-19. Infect Dis (Auckl) . 2020;52(7):517-519. doi:10.1080/23744235.2020.1755447

11. Bongiovanni M, Basile F. Re-infection by COVID-19: a real threat for the future management of pandemia? Infect Dis (Auckl) . 2020;52(8):581-582. doi:10.1080/23744235.2020.1769177

12. Tomassini S, Kotecha D, Bird PW, Folwell A, Biju S, Tang JW. Setting the criteria for SARS-CoV-2 reinfection - six possible cases. J Infect . 2020. doi:10.1016/j.jinf.2020.08.011

13. Keech C, Albert G, Cho I, et al. Phase 1-2 Trial of a SARS-CoV-2 Recombinant Spike Protein Nanoparticle Vaccine. N Engl J Med . 2020;383(24):2320-2332. doi:10.1056/nejmoa2026920

14. Chan PKS, Lui G, Hachim A, et al. Serologic Responses in Healthy Adult with SARS-CoV-2 Reinfection, Hong Kong, August 2020. Emerg Infect Dis . 2020;26(12):3076-3078. doi:10.3201/eid2612.203833

15. Zhang F, Gan R, Zhen Z, et al. Adaptive immune responses to SARS-CoV-2 infection in severe versus mild individuals. Signal Transduct Target Ther . 2020;5(1):156. doi:10.1038/s41392-020-00263-y

\section{Hosted file}

Table_1.pdf available at https://authorea.com/users/414050/articles/522197-sars-cov-2-reinfection-versus-prolonged-shedding-a-case-series

\section{Hosted file}

Figure 1.pdf available at https://authorea.com/users/414050/articles/522197-sars-cov-2-reinfection-versus-prolonged-shedding-a-case-series 